\title{
Meeting Report: AAPS/FDA Workshop on BE, BCS, and Beyond
}

\author{
Vivian A. Gray \\ V.A. Gray Consulting, Inc. \\ Hockessin, DE
}

An AAPS meeting, co-sponsored by FDA, on BCS and BE was held May 21-23, 2007, in North Bethesda, MD. The highlights related to dissolution testing will be given in this report; however, some of the BE (dealing with clinical issues, especially highly variable drug) and permeability aspects will be omitted. All slides can be found on the AAPS website at http://www.aapspharmaceutica.com/ meetings/meeting.asp?id=90. However, these slides will be removed by the first of the year.

\section{Session I: Regulatory Significance of BCS}

The session began with an overview by Janet Woodcock of the FDA. She emphasized that the Critical Path Initiative would further advance the prediction of drug safety and efficacy. This would be obtainable when mechanistic understanding was improved since there is too much guesswork going on presently. Mehul Mehta, also of the FDA, informed the audience about the FDA CDER BCS Committee. This committee provides a point of contact in the FDA for those sponsors who wish to attempt a BCS biowaiver. Lawrence Yu and Mehul Mehta are the co-chairs of the committee. Their goal is to assure consistency and transparency across all therapeutic areas and generic drugs, and to revise the BCS guidance. The committee has reviewed 25 drug products, 16 of which were given BCS Class 1 determinations. Milan Samid of World Health Organization (WHO) discussed the WHO guidelines, specifically Technical Report Series, N. 937,2006, Annex 7 and 8 on interchangeability and BCS. There are some differences between the FDA BCS guidance and the WHO documents, specifically that Class 2 is eligible for biowaiver if the dose-to-solubility ratio at pH 6.8 is $250 \mathrm{~mL}$ or less and high permeability is at $85 \%$ absorbed (instead of $90 \%$ ); and Class 3 is eligible if very rapidly dissolving. James Polli, University of Maryland, spoke on how in vitro studies are sometimes better than conventional human BE studies in assessing equivalence. His main points were that the in vitro studies reduce costs, more directly assess product performance, offer benefits in terms of ethical considerations, and are potentially better if the physician's confidence and understanding can be gained.

\section{Session II: Implementation of BCS and Biowaiver for Class 3 Drugs}

There are an increasing number of successful biowaiver applications, but faster progress could be made if there were international harmonization and the implementation barrier of the perception of risk for project delays could be removed. BCS-based Biowaiver case studies were presented by several industry representatives. Gordon Amidon, University of Michigan, and Salomon Stavchansky, University of Texas at Austin, debated the issue of Biowaivers for Class 3 drugs. One of the points made was that within BCS Class 3, the "risk" of a biowaiver may vary significantly depending on the magnitude of the "poor" $P$ (permeability). Thus, risk analysis techniques may be pertinent to deciding whether or not a BCS 3 biowaiver could be granted. Excipient effects are often cited as a possible source of influence on BA not predicted by dissolution testing, yet there are few examples of common excipients used at typical levels that actually influence $B A B E$ in any significant way. This is a potential area for further research. For example, the "dose-response" effect of excipients could be systematically studied. Another point raised was that there are examples supporting the fact that differences in dissolution of Class 1 compounds with rapid dissolution (within 30 minutes) do not affect $\mathrm{C}_{\max }$ and AUC - possibly there could be a relaxation of the criteria by excluding the requirement for $\mathrm{f}_{2}$ test for Class 1 drugs with complete dissolution within 30 minutes. An unresolved issue was how to handle (from a regulatory standpoint) situations where the formulation may change the BCS class (e. g., from 2 to 1). Since the BCS Class is pegged to the chemical entity, can the regulatory framework accommodate this "formulation" effect? In addition, industry and various individual companies have indicated that a significant majority of new chemical entities are BCS Class 2.Thus, the focus should be on Class 2 rather than on extensions to Class 3, which occur to a relatively low extent. For example, should the regulators be looking at relaxing the Class 1 criteria in a way that would include more Class 2 drugs that "nearly miss" Class 1 ?

\section{Session III: Bioequivalence for Highly Variable and GI Locally Active Drugs \\ Charlie DiLiberti of Barr Labs stressed the importance} of simulations and suggested that $C_{\text {max }}$ variance has little effect on steady state conditions. AUC can have a significant effect, leading him to conclude that $C_{\max }$ and $A U C$ are a better measure of bioequivalence for highly variable drugs. Barbara Davit of FDA pointed out that about 10\% of the BE submissions are for highly variable drugs; of these, the variability in $70 \%$ is attributed to the disposition 
of the drug substance, with the remaining $30 \%$ possibly due to formulation, study conduct, or aberrant subjects. FDA is currently evaluating a scaled average BE approach for analysis of highly variable drugs. Jack Cook, Pfizer, spoke on in vivo-in vitro correlations. He pointed out that Class 2 BCS compounds are amenable to dissolutionbased BE and a significant number would also be amenable to IVIVCs. He recommended that the resources for IVIVC should be based on product variability and undertaken in Phase 2. It was stressed that a dissolution method with a fast dissolution rate would not necessarily be a good predictor of an IVIVC. A physiologically relevant media should be used. A major point was that there are increasing regulatory "requests" to perform IVIVC. Note that in the FDA Manual of Policies and Procedures-Clinical Pharmacology and Biopharmaceutics Review Template (04/27/04), there is the question in section 2.5.5:"How do the dissolution conditions and specifications ensure in vivo performance and quality of the product?" Also, in the FDA Questions to be completed by ANDA Sponsors for the preparation of a QbR-Quality Overall Summary (1/2007), the question is posed in section 2.3.P.5. Control of Drug Product:"What is the drug product specification? What is the justification for the acceptance criterion?" He also pointed out that there are several commercially available software packages that assist the development of IVIVC by simulations and modeling.

\section{Session IV: Biorelevant Dissolution and BCS Future Development}

Paul Dickinson and Bertil Abrahamsson, both from AstraZeneca, addressed the issue of clinical relevance of dissolution testing in quality by design (QbD). The major point they made was that dissolution testing should be applicable to assure desired clinical performance for a wide range of drugs in $\mathrm{QbD}$, based on $\mathrm{BCS}$ considerations and specific product knowledge. Failure to establish a classical IVIVC could be a successful outcome of an IVIV study in context of QbD if all variants (i.e., side batches) produce the same exposure.Variants should be assessed via an in vivo biostudy, and generation of variants should be done during development for the highest risk factor impacting dissolution. Peter Langguth of the Johannes Gulenberg-University Mainz recommended that simulations and modeling may help identity BCS Class 2 biowaiver candidates. There is a need for robust and predictive dissolution methods, combined with additional validation of the simulations. Lawrence Yu of FDA gave a summary of possible revisions to the BCS Class boundaries. A probable revision will change the $\mathrm{pH}$ range from 1-7.5 to 1-6.8 for defining high solubility. Another would be to reduce the permeability boundary from $90 \%$ to $85 \%$. There may be change in the rapid dissolution criteria from
$>85 \%$ in 30 minutes to $>85 \%$ in 45 minutes in the dissolution media of $0.1 \mathrm{~N} \mathrm{HCl}, \mathrm{pH} 4.5$, and $\mathrm{pH} 6.8$ buffers. For Class 2 drugs that are weak acids having low solubility at low $\mathrm{pH}$ and high solubility at high $\mathrm{pH}$, there are concerns about the predictability of in vitro dissolution to in vivo, because there may be incomplete dissolution and the excipients may have an effect on solubility and dissolution. He said that biowaiver extensions to BCS Class 3 drugs were likely, although there is still the concern of excipient effect on permeability (e. g., absorptive transporters and GI motility). He spoke of the two different roles of dissolution. There is the dissolution for in vivo performance, which would be a biorelevant dissolution test that correlates with the in vivo dissolution. This would be a one-time test to provide a baseline for product performance-this is the test that biowaivers would rely on. Then there is the dissolution test for quality control that would be a product-specific test. This test could be the same as the in vivo performance dissolution test. Dissolution conditions would emphasize reproducibility and detection of product changes and would not be constrained to mimic in vivo conditions. The acceptance criteria would be to ensure consistency of the batches. He pointed out that the WHO has proposed a simplified test for solid oral dosage forms that contain highly soluble drugs that would use the paddle at $75 \mathrm{rpm}, 500 \mathrm{~mL}, 37^{\circ} \mathrm{C}$, 30-min sample, and a dissolution media using simulated intestinal fluid at $\mathrm{pH}$ 6.8. The specification would be $\geq 85 \%$ released in 30 minutes.

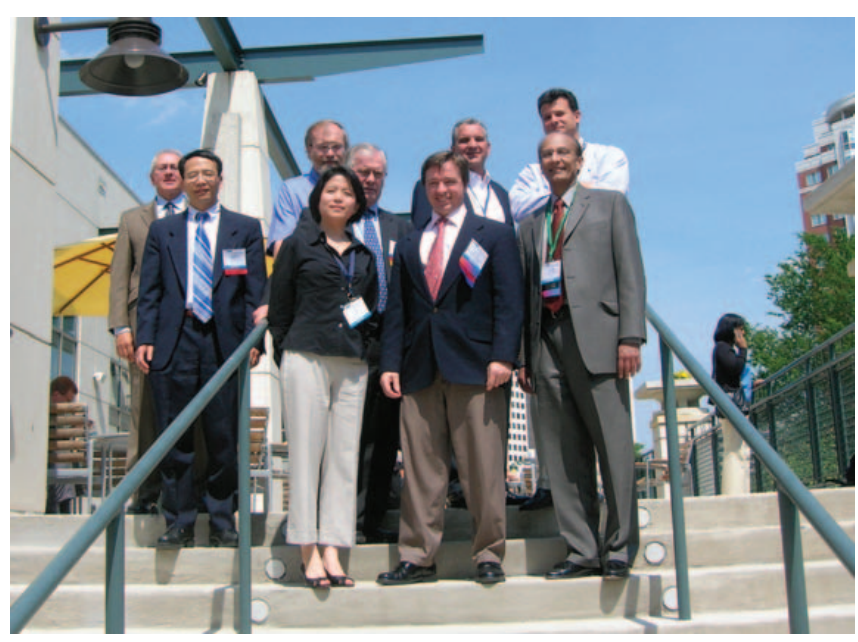

Organizers of the BCS Workshop. Front: Lawrence Yu, Hua Zhang, James Polli, Waseem Malick

Back: Gordon Johnston, Jack Cook, Gordon Amidon, Kerry “Gus" Hartauer, Steve Krill 